\title{
Massive gastrointestinal bleeding caused by a gastrointestinal stromal tumour of the third part of the duodenum treated by means of emergency partial duodenal resection
}

\author{
Grzegorz Jarczyk, Łukasz Bereziak, Marek Jackowski \\ Department of General, Gastroenterological, and Oncological Surgery, Collegium Medicum in Bydgoszcz, Nicolaus Copernicus \\ University in Torun, Poland
}

Address for correspondence: Łukasz Bereziak MD, 89D/5 Bartkiewiczówny St, 87-100 Torun, phone: +48 783689434 , e-mail: lukasz.bereziak@gmail.com

The gastrointestinal stromal tumour (GIST) is a carcinoma derived from precursor stem cells, which differentiate into pacemaker intramural neural cells (Cajal's cells). Once classified as leiomyoma, leiomyoblastoma, leiomyosarcoma, or Schwannoma, it becomes a separate disease entity and the most common sarcoma of the gastrointestinal tract. The above-mentioned tumours are mainly found in the stomach (40-70\%) and small bowel (20-50\%), less frequently in the colon and oesophagus $(<5 \%)$, and rarely outside the gastrointestinal tract (omentum, mesentery, retroperitoneal space).

The incidence rate is estimated at 3-16 cases $/ 1 \mathrm{mln}$ inhabitants, yearly. The presumed number of new cases in Poland is approximately 600 per year [1]. The carcinoma is usually diagnosed in patients after the age of 50 years, more often in the male population. Tumours small in size are asymptomatic. Those located submucosally may be the cause of chronic or subacute, rarely, massive gastrointestinal bleeding [2-5].

The most effective method of treating GIST is radical surgery. Due to the complex duodeno-pancreatic anatomy, a variety of surgical techniques have been proposed in the management of duodenal stromal tumours, from wedge resections to radical pancreatoduodenectomy, depending on the location and size of the tumour [5-12].

The Authors presented a case of radical surgery, considering a massively bleeding duodenal stromal tumour during an emergency shift, which consisted of segmental duodenectomy.

A 45-year-old male patient was directed to the Department of Surgery during an emergency shift, due to massive gastrointestinal bleeding. Bleeding symptoms, including melena and haematemesis, appeared several hours before the hospitalisation. The patient was pale and weak, without a history of coexisting diseases, blood pressure (BP) 100/60 mm Hg, heart rate (HR) $140 /$ min. Prior to gastroscopy, conservative treatment was initiated and blood samples were collected. Since the patient was conscious and cooperative, endoscopy was performed under local anaesthesia assisted by an anaesthesiologist. A large amount of blood was found in the stomach and duodenum, and after its partial aspiration, in the third part of the duodenum we revealed the presence of a large submucosal tumour with a centrally located bleeding ulceration, Forrest $\mathrm{lb}$. An adrenalin solution 1: 10000 was injected into the ulceration, and the bleeding was stopped (Figure 1).

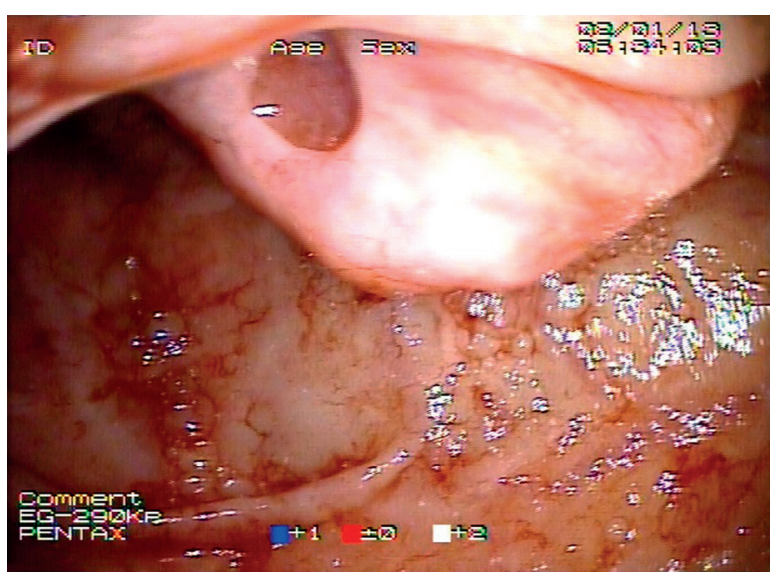

Figure 1. Tumor after endoscopic adrenaline injection 
Conservative treatment was continued administering fluids, proton pump inhibitors, and three units of packed red blood cells. On admission, the haemoglobin level was $5.7 \mathrm{~g} \%$, and biochemistry results were within normal limits. The patient admitted that 6 years earlier he was hospitalised because of upper gastrointestinal bleeding at another medical centre. Gastroscopy was then performed, which revealed the presence of a $3-\mathrm{cm}$ lesion, duodenal tumour with a bleeding ulcer. Bleeding was stopped by means of an adrenalin injection. The collected samples revealed the following - mucosa duodeni normalis, Helicobacter pylori (-). Further treatment was not carried out.

After initial improvement in the patients' general condition, $2 \mathrm{~h}$ after endoscopy his condition deteriorated, vomiting of a large amount of blood was observed. Symptoms of haemorrhagic shock persisted (low blood pressure and increased HR). The decision concerning emergency surgery was undertaken, and another 3 units of packed red blood cells were crossed. During the surgical procedure a tumour located in the third part of the duodenum was visualised, $4 \mathrm{~cm}$ in diameter. The tumour was encapsulated, covering the entire duodenum and expanding towards the intraperitoneal space. The tumour did not infiltrate surrounding tissues, the head of the pancreas, unciform process, or colon mesentery. Metastatic lesions to the liver and peritoneum were not observed (Figure 2).

After incision of the right side of the gastro-transverse ligament and Kocher's manoeuvre, the second, third, and fourth parts of the duodenum to the level of the upper mesenteric vessels were unveiled. The pancreatoduodenal vascular branches were ligated near the duodenal wall, creating the third part of the duodenum from its descending part to the superior mesenteric vein. In the proximal and distal part of the duodenum,

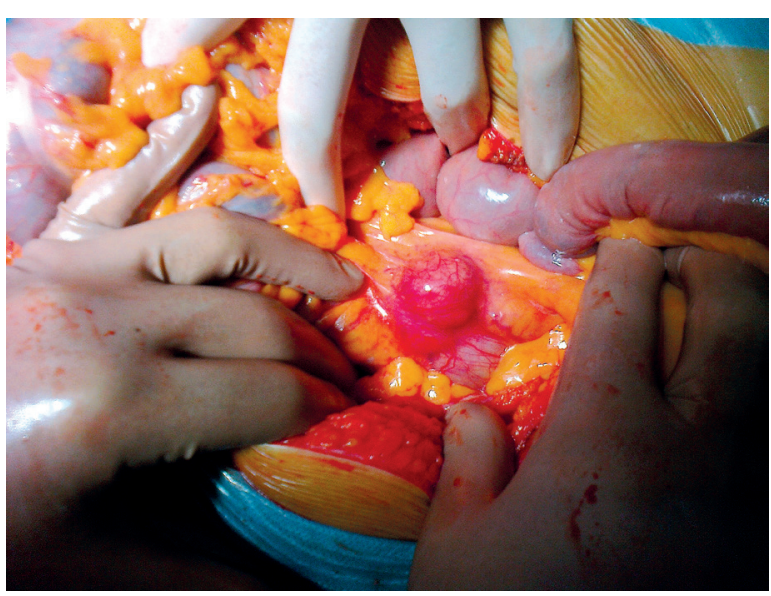

Figure 2. Tumor
2.5 and $1.5 \mathrm{~cm}$ from the tumour, respectively, supporting sutures were applied. The third part of the duodenum together with the tumour and $1 \mathrm{~cm}$ margins were excised. Gastrointestinal continuity was restored after suturing of the descending and ascending part of the duodenum by end-to-end anastomosis, using a two-layered, continuous PDS 3.0 suture (Figure 3). During the procedure the capsule was untouched. The postoperative course was uneventful, and the patient, in good general condition, was discharged from the hospital 7 days after surgery.

The histopathological examination revealed the presence of a solid, gastrointestinal stromal tumour, without necrosis, $4 \mathrm{~cm}$ in diameter, delimited from the duodenal wall. The surgical margins were considered as free of neoplastic disease. The GIST was diagnosed with two mitotic figures per 50 high-power field. The immunohistochemical examination showed a positive reaction with antigen $C D 117$, and none with $C D 34$, as well as the presence of the proliferative index Ki67 (+) in $3 \%$ of tumour cells.

One month after surgical treatment, abdominal computer tomography (CT) was performed, showing no other neoplastic foci. The patient continues to be under oncological and endoscopic control, feeling good. Seven and 12 months after surgery, control abdominal, pelvis and chest $\mathrm{CT}$ examinations were performed, as well

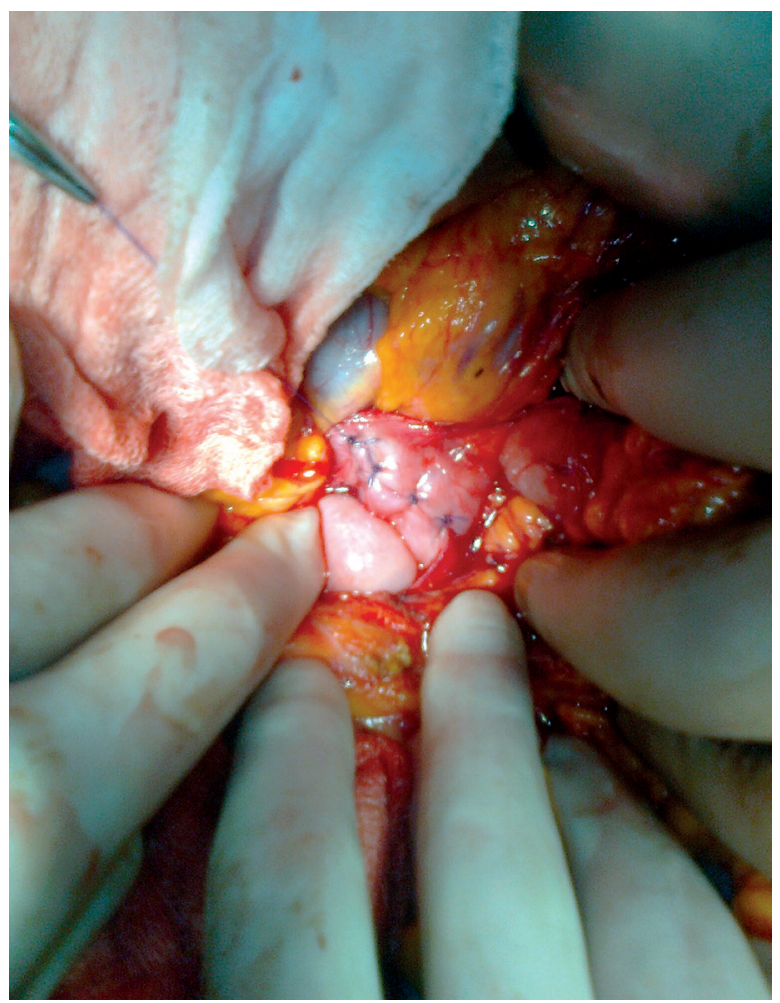

Figure 3. Anastomosis after tumor excision 
as endoscopy. Local recurrence or metastasis was not observed. According to the NCCN-AFIP-AJCC (National Comprehensive Cancer Network - Armed Forces Institute of Pathology - American Joint Committee on Cancer) criteria, determining the degree of aggressiveness of the tumour on the basis of its size and location, and number of mitotic figures [1], the risk of recurrence and metastases was assessed as low. Therefore, the patient was not qualified for targeted, imatinib treatment (low molecular tyrosine kinase inhibitor).

Most gastrointestinal stromal tumours arise sporadically, and are caused by activating mutations in genes encoding KIT membrane receptors (tyrosine kinase) (85\%), and less frequently platelet-derived growth factor, $\alpha$ receptor (PDGFRA) (5\%). In the remaining $10 \%$ wild type GIST can be seen. Mutations of the KIT proto-oncogene lead to the overexpression of the membrane KIT receptor, which becomes active without the need to bond to the ligand. This leads to continuous KIT enzyme activity and uncontrolled cellular growth (lack of adhesion and differentiation). Excessive expression of the KIT membrane receptor is confirmed by immunohistochemical tumour examinations. CD117 antigens combine with the KIT protein giving a colour reaction visible under the microscope [1]. Sampling of the histological material by means of endoscopy is often ineffective, due to the intramural character of the lesion, and may also initiate and exacerbate bleeding from the tumour. In the case of percutaneous biopsy there is the potential risk of pseudocapsule tumour damage and intraperitoneal dissemination [7]. Biopsy under endoscopic ultrasound control seems to be the method of choice in the case of easily accessible submucosal tumours. Percutaneous biopsy is proposed only when the result will affect the decision concerning treatment, when the tumour is unresectable or borderline, and when molecular therapy is planned. The GIST grow intramurally: usually in the muscular, subserous, and submucosal layers, surrounded by a pseudocapsule, reaching a size of 1 to $30 \mathrm{~cm}$. Tumours usually grow towards the peritoneal cavity; hence, the delayed symptoms when the tumour reaches a larger size (pain, intestinal obstruction) [9]. Tumour growth may be exophytic or endophytic, as it was in our case. The GIST exceptionally metastasizes to local lymph nodes (<3\%) [1]. Metastases are usually observed in the liver, more rarely in the lungs and bones. Stromal tumours penetrating the submucous membrane might be responsible for gastrointestinal bleeding. Haemorrhage is often chronic or subacute (anaemia, melena) [2, 3, 6]. Massive bleeding, usually from ulceration at the apex of the tumour is rarely observed $[4,5]$. The authors encountered in literature data only one case of massive bleeding from a stromal duodenal tumour, which after ineffective endoscopic treatment required emergency surgical intervention due to recurrent bleeding episodes and haemorrhagic shock symptoms. Bleeding was stopped by means of ligating blood vessels supplying the tumour. Radical surgery (segmental resection of the third part of the duodenum) was delayed, being performed in a reference centre [5].

In our case, the difficult decision concerning emergency surgery was undertaken, due to the direct life-threatening condition of the patient. The continuation of conservative treatment was considered as uncertain. The decision was all the more difficult, since the patient was in haemorrhagic shock and had to be operated under emergency shift conditions, at night, with limited knowledge as to the size of the tumour and its relation to the pancreatic head. The experience of the endoscopist enabled him to assume the correctness and effectiveness of the primary endoscopic intervention and its probable meaningless continuation in case of bleeding recurrence. The experience of the surgeon enabled him, in case of an infiltrating tumour, to perform pancreatoduodenectomy.

The most effective method of treating GIST is surgery, consisting of excision of the tumour with healthy tissue margins (resection R-0). Since the above-mentioned tumours do not metastasize to lymph nodes, local lymphadenectomy is not required. During the operation one should avoid damage to the pseudocapsule and tumour rupture, which leads to intraperitoneal dissemination [1]. In the case of duodenal GIST, the type and extent of the operation will depend on the size and location of the tumour. Tumours located in the vicinity of Vater's papilla, infiltrating the pancreatic head or uncinate process, may only be treated by means of pancreatoduodenectomy, including pancreatoduodenectomy with pylorus preservation [2]. In the case of tumours located near the papilla, pancreas-preserving duodenectomy may be performed [10]. These radical, extensive procedures are burdened with the risk of complications, even greater than in the case of pancreatoduodenectomy, due to pancreatic head carcinoma. Usually in the case of GIST the pancreatic and biliary ducts are narrow, thus, the risk of postoperative fistula development is greater [7]. Small and medium-sized duodenal tumours, those unrelated to Vater's papilla, might be subjected to wedge resections $[2,9]$ or segmental duodenectomy $[6,11]$. In the latter case the type of reconstruction will depend on the location of the tumour.

Segmental duodenectomy (R-0 resection), due to the small risk of lymph-node metastases, is a justified alternative to pancreatoduodenectomy, giving a comparable oncological efficacy and significantly lower risk of complications $[7,8]$. It is the method of choice consider- 
ing emergency surgery. In our case the tumour did not infiltrate surrounding tissues, but its size and the fact that it entirely covered the duodenum excluded wedge resection. During preparation and devascularisation of the third part of the duodenum, special care was taken to not damage the tumour capsule and the head and uncinate process of the pancreas. Extensive Kocher's manoeuvre enabled us to close and anastomose the well-perfused ends of the second and ascending part of the duodenum. The above-mentioned procedure was the shortest but most radical, which in the case of a patient in shock was of significant value. An alternative method of treatment is anastomosis of the first jejunal loop with the descending part of the duodenum. Duodenoenterostomy is usually performed by means of the end-to-end method [6, 7], or end-to-side, as well as side-to-side methods, after previous depression of the stump of the descending part of the duodenum [8]. End-to-end anastomosis might compromise the patency of Vater's papilla, due to the close vicinity of the suture [5]. When the tumour refers to the duodenal bulb or the part of the duodenum located above Vater's papilla, segmental bulbar and upper descending duodenectomy is performed. After depression of the peripheral part of the duodenum the proximal part of the bulb is anastomosed to the first jejunal loop - Rouxen-Y pylorus-sparing reconstruction [11]. The described segmental resections used in case of GIST are fully accepted. Isolated local recurrence is rarely observed $(<15 \%)[1]$.

The most important prognostic factors after primary tumour excision include the mitotic index, tumour size and location, surgical margins, and possible intraoperative tumour rupture. Increased risk of recurrence and metastases occurs when the lesion is located outside the stomach, its size exceeds $5 \mathrm{~cm}$, and the number of mitoses > 5 [1]. Overall, in $50 \%$ of patients subjected to surgical treatment alone, one may observe local or distant recurrence during the initial 2 years. According to 2009 NCCN guidelines (National Comprehensive Cancer Network), control abdominal and pelvis CT examinations should be performed every 3-6 months during the first 3-5 years, and once every year thereafter. Effective GIST imaging techniques include contrast computer tomography, endoscopy, and ultrasound endoscopy. The MRI is recommended prior to planned rectal GIST surgery, for distant metastases evaluation (to the brain and spinal cord) or when CT is contraindicated. Positron emission tomography is used to diagnose distant metastases and evaluate the effectiveness of imatinib treatment [1].

In case of low-aggressive GIST, control examinations may be performed once a year [1]. Because, even in these patients, recurrence is possible (8\%), life-time surveillance is recommended [5].

In case of advanced gastrointestinal stromal tumours (non-resectable lesion, recurrence after surgery, liver metastases, intraperitoneal dissemination) the use of low-molecular tyrosine kinase inhibitors (imatinib) is the therapeutic method of choice. The GIST is resistant to conventional chemotherapy, and the role of radiotherapy remains undetermined [1].

A bleeding duodenal GIST is a life-threatening condition. Endoscopic control of the bleeding seems to be ineffective. In case of massive bleeding, emergency surgery seems to be the method of choice.

\section{Conflict of interest}

The authors declare no conflict of interest.

\section{References}

1. Rutkowski P, Kulig J, Krzakowski M, et al. Recommendations for diagnostics and therapy of gastrointestinal stromal tumors (GIST). Nowotwory Journal of Oncology 2011; 61: 70-80.

2. Liu S, Zheng Q, Song Z, et al. Gastrointestinal stromal tumor of duodenum: a cause of upper gastrointestinal hemorrhage. Chinese-German J Clin Oncol 2010; 9: 243-5.

3. Köklü S, Filik L, Oztürk ZA, et al. A rare cause of massive gastrointestinal bleeding in a young patient. Dig Liver Dis 2006; 38: 862-3.

4. Giesler T, Müller S, Schmiedehausen K, et al. Bleeding stromal tumor. Gastrointest Endosc 2005; 61: 593.

5. Mennigen R, Wolters H, Schulte B, et al. Segmental resection of the duodenum for gastrointestinal stromal tumor (GIST). World J Surg Oncol 2008; 6: 105.

6. Chung JC, Chu CW, Cho GS, et al. Management and outcome of gastrointestinal stromal tumors of the duodenum. J Gastrointest Surg 2010; 14: 880-3.

7. Bourgouin S, Hornez E, Guiramand J, et al. Duodenal gastrointestinal stromal tumors (GISTs): arguments for conservative surgery. J Gastrointest Surg 2013; 17: 482-7.

8. Buchs NC, Bucher P, Gervaz P, et al. Segmental duodenectomy for gastrointestinal stromal tumor of the duodenum. World J Gastroenterol 2010; 16: 2788-92.

9. Yildırgan MI, Bafloğlu M, Atamanalp SS, et al. Duodenal stromal tumor: report of a case. Surg Today 2007; 37: 426-9.

10. Ravoire A, Poussier M, Facy O, et al. Multiple duodenal stromal tumors revealing type 1 neurofibromatosis: an indication for pancreas-preserving duodenectomy. Surgery 2013; 153: 599-600.

11. Asakawa M, Sakamoto Y, Kajiwara T, et al. Simple segmental resection of the second portion of the duodenum for the treatment of gastrointestinal stromal tumors. Langenbeck's Arch Surg 2008; 393; 605-9.

12. Kazanowski M, Agrawal AK, Zawalski H, et al. An unusual case presentation of a palpable abdominal wall mass: extragastrointestinal stromal tumor with literature review. Prz Gastroenterol 2013; 8: 138-41.

Received: 26.05 .2014

Accepted: 1.10 .2014 